\title{
Perfil epidemiológico de recém-nascidos internados em Unidades de Terapia Intensiva Neonatal em hospitais universitários no extremo Sul do Brasil
}

\author{
Tatiane Britto da Silveira ${ }^{\mathrm{a}}$, Ronan Adler Tavella ${ }^{\mathrm{a}, \mathrm{b}}$, Jullya Brum Fernandez, \\ Ana Paula Foletto Ajalla Ribeiro ${ }^{c}$, Edariane Menestrino Garcia ${ }^{b}$, \\ Flavio Manoel Rodrigues da Silva Júnior ${ }^{\mathrm{a}, \mathrm{b}, \mathrm{d}^{*}}$
}

aPrograma de Pós-Graduação em Ciências da Saúde - Faculdade de Medicina, Universidade Federal do Rio Grande FURG, Rio Grande, RS, Brasil

bLaboratório de Ensaios Farmacológicos e Toxicológicos - LEFT - Instituto de Ciências Biológicas, Universidade Federal do Rio Grande - FURG, Rio Grande, RS, Brasil

${ }^{c}$ Faculdade de Medicina, Universidade Federal do Rio Grande - FURG, Rio Grande, RS, Brasil

${ }^{\mathrm{d} C e n t r o}$ Regional para Estudos, Prevenção e Recuperação de Dependentes Químicos, Universidade Federal do Rio Grande - FURG, Rio Grande, RS, Brasil

Histórico do Artigo
Recebido em:
08/01/2020
Aceito em:
07/03/2020
Palavras-chave:
Neonatologia;
prematuridade;
Unidades de Terapia
Intensiva; baixo peso
ao nascer

ao nascer

\section{Keywords:}

Neonatology;

prematurity;

Intensive Care Units; low birth weight

\begin{abstract}
RESUMO
O objetivo do estudo foi avaliar o perfil epidemiológico de recém-nascidos de Unidades de Terapia Intensiva Neonatal (UTIn) internados entre julho de 2017 e junho de 2018 de três hospitais universitários no extremo Sul do Brasil. Foi realizado um estudo observacional, prospectivo, descritivo e quantitativo, através da análise de prontuários médicos de UTIn de três hospitais universitários na cidade de Pelotas (2 hospitais) e Rio Grande, RS (1 hospital). Os dados coletados continham informações socioeconômicas e demográficas, condições de saúde materna, desfechos relativos ao parto e aos recém-nascidos (RNs) e a comparação entre os hospitais foi realizada através do teste Qui-quadrado, ou exato de Fisher, considerando o valor de $\mathrm{p} \leq 0,05$. Dentre as variáveis maternas analisadas, aquelas que tiveram diferenças significativas entre os hospitais foram: cor da pele, viver com companheiro, tipo de casa, doenças maternas ou em outros filhos, realização de pré-natale o uso de drogas durante a gestação. Já quanto as variáveis dos RN que apresentaram diferenças significativas entre os hospitais foram: baixo peso ao nascer, prematuridade, perímetro cefálico e doenças infecciosas no RN. O conhecimento do perfil epidemiológico de RNs oriundos de UTIn é de fundamental importância para o gerenciamento destas unidades de terapia intensiva, bem como para o acompanhamento mais efetivo dos egressos nestas Unidades.
\end{abstract}

Epidemiological profile of newborns admitted to Neonatal Intensive Care Units in university hospitals in the extreme south of Brazil

\section{ABSTRACT}

The aim of this study was to evaluate the epidemiological profile of newborns from Neonatal Intensive Care Units (nICU) admitted between July 2017 and June 2018 of three university hospitals in the extreme south of Brazil. An observational, prospective, descriptive and quantitative study was conducted through the analysis of nICU medical records of three university hospitals in the city of Pelotas ( 2 hospitals) and Rio Grande (1 hospital), RS. The data collected contained socioeconomic and demographic information, maternal health conditions, outcomes related to childbirth and newborns (NBs) and the comparison between hospitals was performed using the Chi-square test, or Fisher exact test, considering the value of $\mathrm{p} \leq 0.05$. Among the maternal variables analyzed, those that had differences between hospitals were: skin color, living with a partner, type of house, maternal or other siblings diseases, prenatal care, and drug use during pregnancy. As for the newborn variables, those that had differences between hospitals were: low birth weight, prematurity, head circumference and infectious diseases in the newborn. Knowledge of the epidemiological profile of newborns from nICU is of fundamental importance for the management of these intensive care units, as well as for the more effective monitoring of the egresses in these units.

\footnotetext{
*Autor correspondente: f.m.r.silvajunior@gmail.com (Silva Júnior F.M.R.)
} 


\section{Introdução}

O período neonatal, compreendido entre o nascimento e os primeiros 28 dias de vida, é um momento de grande vulnerabilidade na vida de um recém-nascido, onde se concentram riscos biológicos, ambientais, socioeconômicos e culturais (1). Este período constitui o principal componente da mortalidade infantil mundial (2), no qual a maior parte dessas mortes está relacionada à prematuridade, asfixia e infecções. Além disso, e a partir de dados obtidos pelo Ministério da Saúde em 2012, estima-se que cerca de $25,0 \%$ das mortes neonatais acontecem nas primeiras vinte e quatro horas de vida (3).

Grande parte das mortes neonatais são consideradas evitáveis. Isso acontece, pois o componente neonatal possui relação estreita com as condições de atenção à saúde da mulher durante a gestação, e também de acesso aos serviços de atenção ao parto e ao nascimento (3). Nesse sentido, uma atenção qualificada, é fundamental tanto para saúde materna quanto para o neonato.

Frente a essa necessidade existem as Unidades de Terapia Intensiva Neonatal (UTIn). Essa unidade é um serviço de internação responsável pelo cuidado integral ao recémnascido grave ou potencialmente grave, dotado de estruturas assistenciais que possuam condições técnicas adequadas à prestação de assistência especializada (4). A UTIn oferece um ambiente apropriado para a detecção de situações de risco, com equipamentos diversificados e grande aporte tecnológico, bem como profissionais multidisciplinares capacitados para prestar um cuidado seguro (5). Dentre as principais patologias encontradas na UTIn estão a prematuridade e baixo peso ao nascer, o distúrbio da membrana hialina e a síndrome do desconforto respiratório (6).

Apesar da existência das UTIn, a assistência materno-infantil muitas vezes tem sido considerada insuficiente a nível nacional, existindo ainda grande distância entre o preconizado pelas políticas públicas de saúde e a vida real. Resultando, assim, na inadequação da assistência desde os níveis de atenção mais básicos até os níveis de maior complexidade estrutural, a exemplo da atenção prestada nas UTIn (7).

A disponibilização de dados sobre a população atendida nos serviços hospitalares, em especial nas UTIn possibilita a construção de dados demonstrativos da realidade e necessidades enfrentadas, orientando, enfim, o planejamento de ações nos serviços de saúde. Assim, o conhecimento de dados epidemiológicos de morbimortalidade de uma unidade de saúde permite a tomada de decisões estratégicas visando ao aperfeiçoamento da qualidade de atenção, e buscando planejamentos com vistas à adequação da unidade às características demográficas e de morbidade da população que ela recebe (8).

Frente ao exposto, pressupõe-se que o conhecimento das características maternas e clínicas dos recém-nascidos admitidos na UTIn podem auxiliar no desenvolvimento de políticas que possibilitem uma melhor assistência à saúde e a organização do cuidado materno-infantil, bem como desenvolver intervenções preventivas e terapêuticas apropriadas ao público estudado, tanto regional, quanto nacionalmente. Assim, o objetivo desta pesquisa foi avaliar o perfil epidemiológico de recém-nascidos de Unidades de Terapia Intensiva Neonatal internados entre julho de 2017 e junho de 2018 de três hospitais universitários do extremo Sul do Brasil.

\section{Materiais e Métodos}

Esta pesquisa foi realizada sob os auspícios do Comitê de Ética em Pesquisa na Área da Saúde (CEPAS), da Universidade Federal do Rio Grande (FURG), e recebeu o número de parecer 50/2017 (CEPAS/FURG). Ademais, o Termo de Consentimento Livre e Esclarecido foi obtido de todos responsáveis pelos RN após a explicação dos 
objetivos, metodologias, benefícios e riscos do estudo. Todas as informações que poderiam ser usadas para identificar participantes individuais estão sendo mantidas no "Laboratório de Ensaios Farmacológicos e Toxicológicos" da FURG na cidade de Rio Grande, localizada no estado do Rio Grande do Sul, Brasil.

Este estudo prospectivo foi conduzido com dados obtidos de 3 UTIs Neonatais durante o período de 12 meses. Participaram do estudo um total de 254 RNs que foram internados nas UTIns de três hospitais localizados nos municípios de Pelotas (Hospital Universitário da Universidade Católica de Pelotas - HU/UCPEL, e Hospital Escola da Universidade Federal de Pelotas - HE/UFPEL) e de Rio Grande (Hospital Universitário da Universidade Federal do Rio Grande - HU/FURG). Como critério de inclusão para compor a amostra da pesquisa adotou-se recém-nascidos admitidos vivos na UTI neonatal dos 3 hospitais avaliados, no período de julho de 2017 a junho 2018, cujo os responsáveis assinaram o termo de consentimento livre e esclarecido. Como critério de exclusão, adotou-se todos aqueles que não se enquadraram nos critérios de inclusão.

A coleta de dados se deu através de um instrumento elaborado pelos pesquisadores constando dados maternos e dados do $\mathrm{RN}$ oriundos dos prontuários médicos, após assinatura do Termo de Consentimento Livre e Esclarecido por algum responsável pelo RN. As variáveis coletadas e apresentadas da seguinte forma: variáveis socioeconômicas e demográficas - idade ( $<18$ anos ou $\geq 18$ anos), cor da pele (branca ou não branca), viver com companheiro (não ou sim), escolaridade (até 8 anos e acima de 8 anos), tipo de habitação (alvenaria ou outros); condições de saúde - doenças maternas (não ou sim), doenças em outros filhos (não ou sim), abortos espontâneos prévios (não ou sim), número de abortos espontâneos (1 ou 2 ou mais); informações da gestação - realização de pré-natal (não ou sim), número de consultas de pré-natal ( $<6$ ou $\geq 6$ ), uso de álcool, cigarro e drogas ilícitas durante a gestação (não ou sim); condições do recém-nascido - gênero (feminino ou masculino), peso ao nascer $(<2500$ $\mathrm{g}$ ou $\geq 2500 \mathrm{~g}$ ), idade gestacional ( $<37$ semanas ou $\geq 37$ semanas), perímetro cefálico $(<32 \mathrm{~cm} \mathrm{ou} \geq 32 \mathrm{~cm})$; APGAR ao primeiro minuto ( 0 a 6 ou 7 a 10$)$, APGAR ao quinto minuto (0 a 6 ou 7 a 10), malformações congênitas, doenças infecciosas, infecções sexualmente transmissíveis e doenças respiratórias (não ou sim). As análises estatísticas foram realizadas no software PRISM $5.0 \mathrm{com}$ a associação entre as variáveis sendo realizada pelo teste do Qui-quadrado ou teste Exato de Fisher, considerando um $\mathrm{p}$ significativo $\leq 0,05$.

\section{Resultados}

Os dados referentes às variáveis socioeconômicas e demográficas coletadas no estudo estão dispostos na Tabela 1. Diferenças significativas entre os 3 hospitais foram apontadas nas variáveis cor da pele, viver com companheiro e tipo de habitação. Enquanto o hospital 1 teve um percentual majoritariamente de mães de cor de pele branca $(84 \%)$, os outros dois tinham, aproximadamente um terço das mães de cor da pele não-branca. O hospital 1 teve o percentual de $90 \%$ de mães que vivem com companheiro, enquanto o hospital 2 o percentual foi de $57 \%$ e no hospital 3 foi de $39 \%$. Enquanto o percentual de casas de alvenaria nos hospitais 1 e 2 foi superior a $80 \%$, no hospital 3 o percentual foi de $67 \%$ (Tabela 1 ). 
Tabela 1 - Variáveis socioeconômicas e demográficas das mães de RNs internados nas UTIn dos hospitais universitários estudados.

\begin{tabular}{|c|c|c|c|c|}
\hline & $\begin{array}{c}\text { HOSPITAL } 1 \\
(\mathrm{~N}=146)\end{array}$ & $\begin{array}{c}\text { HOSPITAL } 2 \\
(\mathrm{~N}=65)\end{array}$ & $\begin{array}{c}\text { HOSPITAL } 3 \\
(\mathrm{~N}=43)\end{array}$ & VALOR DE P \\
\hline IDADE (ANOS) & & & & 0,17 \\
\hline$<18$ & $6 \%(8)$ & $10 \%(6)$ & $14 \%(6)$ & \\
\hline$\geq 18$ & $94 \%(136)$ & $90 \%(56)$ & $86 \%(36)$ & \\
\hline COR DA PELE & & & & $\mathbf{0 , 0 2}$ \\
\hline BRANCA & $84 \%(87)$ & $71 \%(41)$ & $68 \%(21)$ & \\
\hline NÃO BRANCA & $16 \%(17)$ & $29 \%(17)$ & $32 \%(10)$ & \\
\hline $\begin{array}{l}\text { VIVE COM } \\
\text { COMPANHEIRO }\end{array}$ & & & & $<0,0001$ \\
\hline NÃO & $10 \%(12)$ & $43 \%(18)$ & $61 \%(19)$ & \\
\hline SIM & $90 \%$ (107) & $57 \%(42)$ & $39 \%(12)$ & \\
\hline $\begin{array}{l}\text { ESCOLARIDADE } \\
\text { (ANOS) }\end{array}$ & & & & 0,09 \\
\hline ATÉ 8 ANOS & $50 \%(59)$ & $35 \%(17)$ & $40 \%(12)$ & \\
\hline$>8$ ANOS & $50 \%(58)$ & $65 \%(32)$ & $60 \%(18)$ & \\
\hline $\begin{array}{l}\text { TIPO DE } \\
\text { HABITAÇÃO }\end{array}$ & & & & $<0,0001$ \\
\hline ALVENARIA & $85 \%(50)$ & $94 \%(45)$ & $63 \%(19)$ & \\
\hline OUTROS & $15 \%(9)$ & $7 \%(3)$ & $37 \%(11)$ & \\
\hline
\end{tabular}

Hospital 1: HE/UFPEL

Hospital 2: HU/UCPEL

Hospital 3: HU/FURG

Em relação às condições de saúde, o percentual de mães com alguma patologia (crônica ou aguda) no hospital 1 foi de $76 \%$, enquanto nos hospitais 2 e 3 foi de aproximadamente $40 \%$. Por outro lado, a prevalência de patologias em outros filhos, entre as mães do hospital 1 foi de 1\%, enquanto nos hospitais 2 e 3 foi de 6 e $12 \%$, respectivamente (Tabela 2). O percentual de mães que realizaram o pré-natal diferiu significativamente entre os 3 hospitais. Enquanto o percentual de mães que não realizaram o pré-natal no hospital 1 foi de $2 \%$, o percentual chegou a $10 \%$ entre as mães do hospital 3. Ainda assim, entre as mães que realizaram o pré-natal, o número de consultas satisfatórias não diferiu significativamente entre os 3 hospitais estudados. $\mathrm{O}$ uso de tabaco, álcool e outras drogas durante a gestação foi verificado entre $23 \%$ das mães do hospital 1, 9\% do hospital 2 e $17 \%$ do hospital 3 (Tabela 3).

Tabela 2 - Condições de saúde das mães de RNs internados nas UTIn dos hospitais universitários estudados.

\begin{tabular}{|c|c|c|c|c|}
\hline & $\begin{array}{c}\text { HOSPITAL } 1 \\
(N=146)\end{array}$ & $\begin{array}{c}\text { HOSPITAL } 2 \\
(N=65)\end{array}$ & $\begin{array}{c}\text { HOSPITAL } 3 \\
(N=43)\end{array}$ & VALOR DE P \\
\hline $\begin{array}{l}\text { DOENÇAS } \\
\text { MATERNAS }\end{array}$ & & & & $<0,0001$ \\
\hline NÃO & $24 \%(27)$ & $59 \%(20)$ & $58 \%(22)$ & \\
\hline SIM & $76 \%(85)$ & $41 \%(14)$ & $42 \%(16)$ & \\
\hline $\begin{array}{l}\text { DOENÇAS EM } \\
\text { OUTROS } \\
\text { FILHOS }\end{array}$ & & & & 0,006 \\
\hline NÃO & $99 \%(94)$ & $94 \%(33)$ & $88 \%(28)$ & \\
\hline SIM & $1 \%(1)$ & $6 \%(2)$ & $12 \%(4)$ & \\
\hline
\end{tabular}


Continuação

\begin{tabular}{l|cccc}
\hline & $\begin{array}{c}\text { HOSPITAL 1 } \\
(\mathbf{N = 1 4 6 )}\end{array}$ & $\begin{array}{c}\text { HOSPITAL 2 } \\
(\mathbf{N}=65)\end{array}$ & $\begin{array}{c}\text { HOSPITAL 3 } \\
(\mathbf{N}=\mathbf{4 3})\end{array}$ & $\begin{array}{c}\text { VALOR DE P } \\
\text { ABORTOS }\end{array}$ \\
PRÉVIOS & $77 \%(106)$ & $71 \%(39)$ & $81 \%(34)$ & \\
NÃO & $23 \%(32)$ & $29 \%(16)$ & $19 \%(8)$ & \\
SIM & & & \\
NÚMERO DE & & & \\
ABORTOS & $75 \%(24)$ & $75 \%(12)$ & $75 \%(6)$ & \\
1 & $25 \%(8)$ & $25 \%(4)$ & $25 \%(2)$ & \\
2 OU MAIS & &
\end{tabular}

Hospital 1: HE/UFPEL

Hospital 2: HU/UCPEL

Hospital 3: HU/FURG

Tabela 3 - Desfechos gestacionais das mães de RNs internados nas UTIn dos hospitais universitários estudados.

\begin{tabular}{|c|c|c|c|c|}
\hline & $\begin{array}{c}\text { HOSPITAL } 1 \\
(\mathrm{~N}=146)\end{array}$ & $\begin{array}{c}\text { HOSPITAL } 2 \\
(\mathrm{~N}=65)\end{array}$ & $\begin{array}{c}\text { HOSPITAL } 3 \\
(\mathrm{~N}=43)\end{array}$ & VALOR DE P \\
\hline $\begin{array}{l}\text { REALIZOU } \\
\text { PRÉ-NATAL }\end{array}$ & & & & 0,04 \\
\hline NÃO & $2 \%(3)$ & $5 \%(3)$ & $10 \%(4)$ & \\
\hline SIM & $98 \%$ (138) & $95 \%(57)$ & $90 \%(38)$ & \\
\hline $\begin{array}{l}\text { NÚMERO DE } \\
\text { CONSULTAS }\end{array}$ & & & & 0,08 \\
\hline$<6$ & $38 \%(51)$ & $51 \%(29)$ & $37 \%(14)$ & \\
\hline$\geq 6$ & $62 \%(82)$ & $49 \%(28)$ & $63 \%(24)$ & \\
\hline $\begin{array}{l}\text { USO DE } \\
\text { DROGAS } \\
\text { DURANTE A } \\
\text { GESTAÇÃO }\end{array}$ & & & & $\mathbf{0 , 0 2}$ \\
\hline NÃO & $77 \%(95)$ & $91 \%(51)$ & $83 \%(35)$ & \\
\hline SIM & $23 \%(29)$ & $9 \%(5)$ & $17 \%(7)$ & \\
\hline
\end{tabular}

Hospital 1: HE/UFPEL

Hospital 2: HU/UCPEL

Hospital 3: HU/FURG

A Tabela 4 mostra os desfechos desfavoráveis relativos ao parto e aos recém-nascidos oriundos das UTIs neonatal dos 3 hospitais estudados. Os desfechos que apresentaram diferença significativa entre os hospitais foram: baixo peso ao nascer, prematuridade, perímetro cefálico, doenças infecciosas e ISTs. Maior prevalência de baixo peso ao nascer, prematuridade e menor perímetro cefálico foram obtidos entre os RNs dos hospitais 2 e 3, enquanto um percentual superior a $40 \%$ de RNs dos hospitais 1 e 2 tiveram diagnóstico de doenças de origem infecciosa.

Tabela 4 - Variáveis e desfechos relativos aos RNs internados nas UTIn dos hospitais universitários estudados.

\begin{tabular}{l|cccc}
\hline & $\begin{array}{c}\text { HOSPITAL 1 } \\
(\mathbf{N = 1 4 6 )}\end{array}$ & $\begin{array}{c}\text { HOSPITAL 2 } \\
(\mathbf{N = 6 5 )}\end{array}$ & $\begin{array}{c}\text { HOSPITAL 3 } \\
(\mathbf{N = 4 3 )}\end{array}$ & VALOR DE P \\
\hline GÊENRO & & & & 0,06 \\
FEMININO & $41 \%(60)$ & $55 \%(33)$ & $56 \%(24)$ & \\
MASCULINO & $59 \%(86)$ & $45 \%(27)$ & $44 \%(19)$ & \\
PESO & & & & $\mathbf{0 , 0 0 3}$ \\
$<2500 G$ & & & $79 \%(34)$ & \\
$\geq 2500 G$ & $64 \%(94)$ & $84 \%(53)$ & $21 \%(9)$ &
\end{tabular}




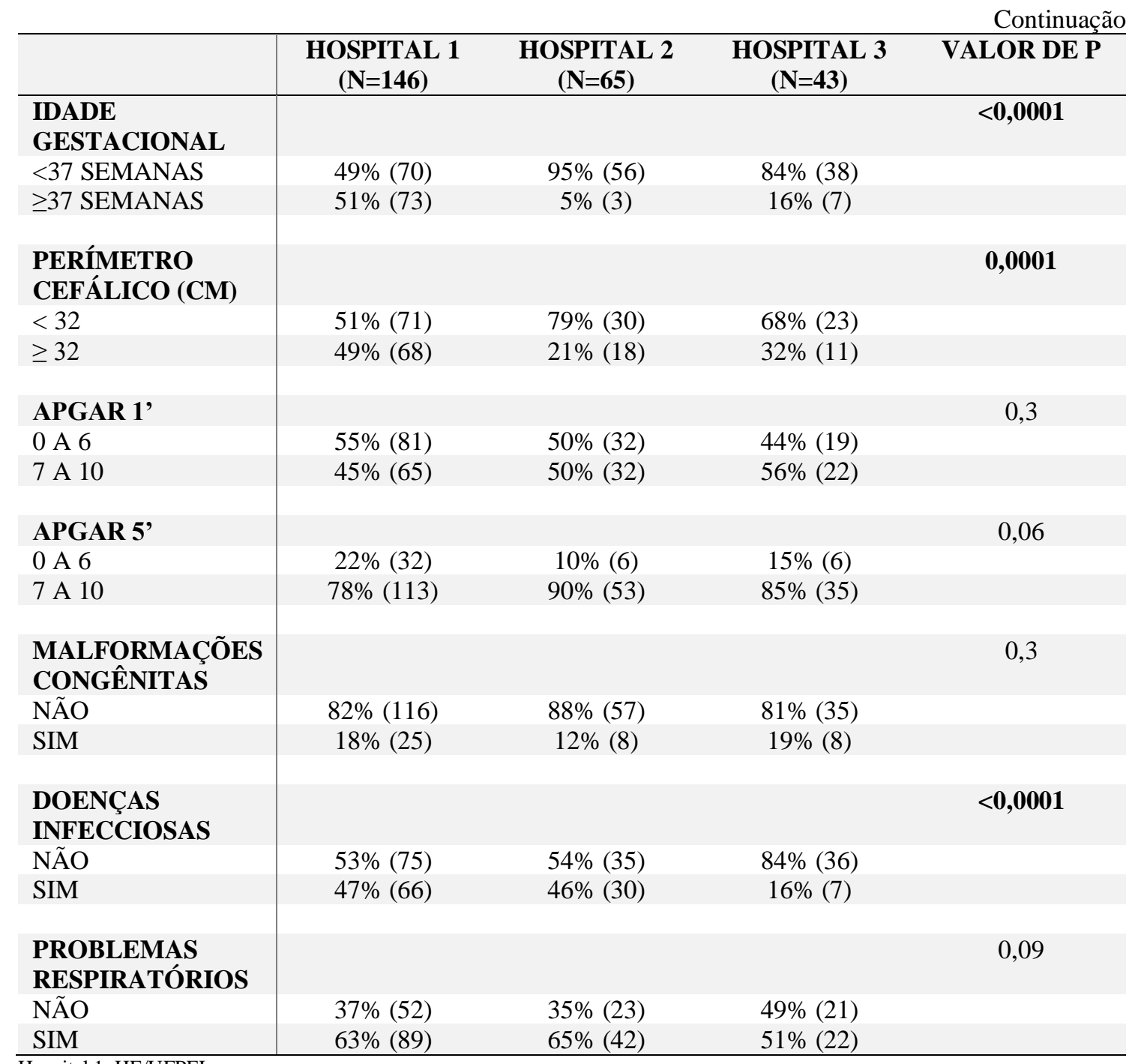

Hospital 1: HE/UFPEL

Hospital 2: HU/UCPEL

Hospital 3: HU/FURG

\section{Discussão}

O presente estudo foi conduzido em Unidades de Terapia Intensiva Neonatal de 3 hospitais universitários situados no extremo Sul do Brasil, sendo dois no município de Pelotas e um no município do Rio Grande. Foram avaliados dados de 254 RNs que internaram nas UTIn durante o período de um ano, bem como informações socioeconômicas, demográficas e de saúde materna. Embora os três hospitais tenham proximidade física, inúmeras variáveis analisadas foram diferentes entre os hospitais, mesmo os situados no mesmo município. Este tipo de estudo é de extrema relevância, pois a análise das condições de nascimento e óbitos de neonatos, informações biológicas da gestação e do parto possibilitam a construção de dados demonstrativos da real qualidade de assistência materno-infantil, orientando o planejamento de ações nos serviços de saúde $(9,10)$.

De maneira geral, o perfil epidemiológico do hospital 1 foi diferente dos outros dois hospitais. As mães dos RNs que internaram no hospital 1 eram predominantemente de cor de pele branca, viviam com companheiro e em casas de alvenaria. Embora a maioria tenha realizado número de consultas pré-natal adequado, foi o hospital onde houve maior relato de uso de drogas durante a gestação, bem como o relato de doenças 
(crônicas ou agudas) maternas. Ainda assim, dentre os hospitais estudados, o hospital 1 possuía o menor percentual de baixo peso ao nascer, prematuridade e de baixo perímetro cefálico. Por outro lado, nos hospitais 2 e 3 a prevalência de doença materna e uso de drogas na gestação foi menor que no hospital 1, mas os desfechos desfavoráveis relativos ao $\mathrm{RN}$ foram mais prevalentes nestes dois hospitais. Nestes dois hospitais, o percentual de mães não-brancas e que vivam sem companheiro foi mais alto que no hospital 1.

Podemos verificar que a prevalência de gestantes que realizaram menos de 6 consultas pré-natal foi alta nos 3 hospitais estudados. Esse dado é importante, pois o mínimo de seis consultas pré-natal, adequadamente distribuídas entre os trimestres da gestação, constitui-se fator de proteção em relação a riscos e agravos materno-infantil e a ausência, ou número inferior, dessas consulta pode impedir que desfechos desfavoráveis para o recém-nascido tenham a intervenção correta por parte dos profissionais da saúde (11).

Em termos de prevalência geral, os principais efeitos adversos entre os RNs das UTIn dos hospitais estudados foram, respectivamente: prematuridade, baixo peso ao nascer, perímetro cefálico reduzido e doenças respiratórias e estes desfechos estão entre os mais prevalentes nesta população $(12,13)$. Estudos tem mostrado que esses desfechos negativos estão diretamente relacionados com possíveis complicações futuras ao recémnascido. Essas complicações podem se apresentar logo nos estágios iniciais de crescimento, como a síndrome de sofrimento respiratório e a hemorragia intraventricular, como também em estágios mais avançados como dificuldades cognitivas e de aprendizagem, entre outras sequelas $(14,15,16)$.

A prevalência de prematuridade e baixo peso ao nascer no presente estudo, notadamente dos hospitais 2 e 3 foi superior aos dados obtidos em estudos da região Sul $(17,18)$ e Centro-Oeste $(19,20)$, mas similares aos obtidos de estudos em Cuiabá-MT (21) e Belém-PA (22). A prevalência de problemas respiratórios e doenças infecciosas também foi superior aos dados obtidos em outros estudos no Sul do Brasil $(17,18)$.

Algumas limitações foram identificadas no presente estudo e devem ser previstas em estudos futuros para melhorar a qualidade da informação. Primeiro, o tipo de coleta realizado, através de prontuários, pode acabar gerando subnotificações aos pesquisadores. Segundo, o número amostral apresentou desigualdades entre os hospitais avaliados, fato decorrente de critérios próprios e condutas de internação diferentes entre os locais de amostragem. Terceiro, dados relativos ao tipo de parto, tempo médio de internação, bem como a taxa de mortalidade, não foram registrados, mas seriam de extrema relevância para um maior panorama do perfil epidemiológico de recémnascidos internados em UTIs neonatais.

Com base no exposto, os dados obtidos contribuem para caracterizar a amostra de recém-nascidos, admitida em serviços de saúde de UTI Neonatal de hospitais universitários do extremo sul do Brasil, revelando as suas principais características epidemiológicas, bem como de suas progenitoras. Ademais, tal estudo também possui como contribuição o direcionamento e planejamento de ações assistenciais voltadas à promoção da saúde materna e neonatal e à minimização de seus agravos.

\section{Conclusão}

A prematuridade, baixo peso ao nascer, perímetro cefálico reduzido e doenças respiratórias foram os desfechos desfavoráveis mais prevalentes entre os RNs internados nas UTIn dos 3 hospitais estudados, mas as variáveis socioeconômicas e demográficas, de condições de saúde e do RN diferiram significativamente entre os 3 hospitais. 
T. B. da Silveira et al./ Vittalle v. 32, n. 2 (2020) 46-54

Ademais, este tipo de estudo possui como principais contribuições o direcionamento, planejamento e fortalecimento de ações assistenciais nos serviços de saúde.

\section{Agradecimentos}

O presente trabalho foi realizado com apoio da Coordenação de Aperfeiçoamento de Pessoal de Nível Superior - Brasil (CAPES) - Código de Financiamento 001. Os autores RAT e TBS receberam bolsa de doutorado da CAPES. A autora JBF recebeu bolsa de Iniciação Científica do CNPq.

\section{Referências}

1. BRASIL. Ministério da Saúde. Secretaria de Atenção à Saúde. Departamento de ações programáticas e estratégicas. Atenção à saúde do recém-nascido: guia para os profissionais de saúde. Vol. 3; Brasília: Ministério da Saúde; 2011.

2. OMS. Organização Mundial da Saúde. Saúde sexual e reprodutiva. Geneva: World Health Organization, 2018. Disponível em: https:/www.who.int/reproductivehealth/global-estimatespreterm-birth/en/.

3. BRASIL, Ministério da Saúde. Secretaria de Vigilância em Saúde. Mortalidade infantil no Brasil: tendências, componentes e causas de morte no período de 2000 a 2010. Brasília: Ministério da Saúde; 2012.

4. BRASIL, PORTARIA No 930, de 10 de maio de 2012; 2012. Disponível em: http://bvsms.saude.gov.br/bvs/saudelegis/gm/2012/prt0930_10_05 2012.html

5. Tomazoni, A, Rocha, PK. Ribeiro MB, Serapião LS, Souza S, Manzo BF. Segurança do paciente na percepção da enfermagem e medicina em unidades de terapia intensiva neonatal. Rev Gaúcha Enferm 2017; 38(1): e64996.

6. Martins EL, Padoin, SMM, Rodrigues AP, Zuge SS, Paula CC, Trojahn TC, Bick MA. Caracterização De Recém-Nascidos De Baixo Peso Internados Em Uma Unidade De Terapia Intensiva Neonatal 2013; 3(1): 155-163.

7. De Lima SS, Da Silva SM, Ávila PES, Nicolau MV, Das Neves PMV. Aspectos clínicos de recémnascidos admitidos em Unidade de Terapia Intensiva de hospital de referência da Região Norte do Brasil. ABCS Health Sci 2015; 40(2): 62-68.

8. Lanetzki CS, De Oliveira CAC, Bass LM, Abramovici S, Troster EJ. O perfil epidemiológico do centro de terapia intensiva pediátrico do hospital israelita Albert Einstein. Einstein 2012; 10(1): 16-21

9. Sharek PJ, Horbar JD, Mason W, Bisarya H, Thurm CW, Suresh G, Classen D. Adverse events in the neonatal intensive care unit: development, testing, and findings of an NICU-focused trigger tool to identify harm in North American NICUs. Pediatrics 2006; 118: 1332-1340.

10. Gesser AGP, Vargas D, Arcoverde TL, Saorim A, Steingräber FA, Stinghen GY, Giacomini G, Hoeller AA. Perfil epidemiológico de recém-nascidos atendidos em uma maternidade de alto risco no Sul do Brasil. VITTALLE; 2019: 31(2), 25-31.

11. Basso CG, Neves ET, Silveira A. Associação entre realização de pré-natal e morbidade neonatal. Texto Contexto Enferm 2012; 21(2): 269-76.

12. Lanzillotti LS, De Seta MH, de Andrade CLT, Junior WVM. Eventos adversos e outros incidentes na unidade de terapia intensiva neonatal. Ciência \& Saúde Coletiva 2015: 20(3): 937-946.

13. Ventura CMU, Alves JGB, do Amaral Meneses J. Eventos adversos em unidade de terapia intensiva neonatal. Rev Bras Enf 2012: 65(1): 49-55.

14. Saigal S, Doyle LW. An overview of mortality and sequelae of preterm birth from infancy to adulthood. The Lancet 2008: 371: 261-9.

15. Vohr B. Speech and language outcomes of very preterm infants. Seminars in Fetal and Neonatal Medicine 2014: 19: 78-83.

16. Singh GK, Kenney MK, Ghandour RM, Kogan MD, Lu MC. Mental health outcomes in US children and adolescents born prematurely or with low birthweight. Depression Research and Treatment 2013: 13. 
17. Cardoso DJDS, Schumacher B. Epidemiological characteristics of neonatal admissions in a public maternity. Rev Enf UFPI 2018; 6(4): 28-32.

18. de Melo Marques G, Pieszak GM, Arrué AM, Rodrigues AP, Gomes GC, Soares R K. Perfil epidemiológico de neonatos de uma unidade de terapia intensiva. Rev Eletr Acervo Saúde 2018; 10(6): 2320-2328

19. Borges FRS, da Silva DR, Matsuy MA, Silva MP, de Carvalho KCN. Perfil epidemiológico de uma unidade de terapia intensiva neonatal em Goiás, Brasil entre 2009 e 2013 Rev Educ Saúde 2016; 4(1): 67-78.

20. Ferraresi MF, da Rocha Arrais A. Perfil epidemiológico de mães de recém-nascidos admitidos em uma unidade neonatal pública. Rev Rede Enf Nordeste 2016; 17(6): 733-740.

21. Reis ACF, Cavalcante SKD, dos Santos ILF. Perfil epidemiológico das infecções hospitalares em uma unidade de terapia intensiva neonatal de um hospital de Cuiabá. TCC-Enfermagem UNIVAG 2018; $1-9$.

22 de Lima SS, da Silva SM, Avila PES, Nicolau MV, das Neves PFM. Aspectos clínicos de recémnascidos admitidos em Unidade de Terapia Intensiva de hospital de referência da Região Norte do Brasil. ABCS Health Sciences 2015; 40(2): 62-68. 